\title{
Potential role of immunological factors in early diagnosis of cancer cachexia in C26 tumor-bearing mice
}

\author{
Jae Eun Ju, ${ }^{1,2}$, Mi-Sook Kim³ ${ }^{3}$ Joo Hyun Kang ${ }^{2}$, Ji Young Lee ${ }^{4}$, Mi So Lee ${ }^{4}$, Eun Ho Kim ${ }^{5}$, Namhyun Chung ${ }^{1}$ \\ and Youn Kyoung Jeong ${ }^{4^{*}}$ (D)
}

\begin{abstract}
Cachexia is a wasting syndrome associated with high mortality in cancer patients through inducing the failure of normal metabolism and reducing the efficacy of cancer treatment. Thus, it is critically important to diagnose cancer cachexia early. To provide background data for the diagnosis of cachexia, cancer cachectic factors were characterized in the present situation, including immunological cachectic changes during cachexia progression in a cancer cachexia mouse model. Major constitution of cachexia progression is known as the stages of pre-cachexia, cachexia, and refractory cachexia. In the pre-cachexia stage, the weights of immune-related organs, including the thymus and spleen were significantly. T cell populations in spleen were markedly reduced and cachectic cytokines consistently increased in a time-dependent manner. Immunosuppression by activation of cytotoxic T-lymphocyte-associated antigen 4 was induced earlier in $\mathrm{CD}^{+}$cells versus other T cell populations. Furthermore, monocyte chemoattractant protein 1 and interleukin-6 levels in the cachexia group were significantly increased at 3 days from C26 cell inoculation whereas significant carcass weight loss as a classical diagnostic marker occurred at 9 days from C26 cell inoculation. In conclusion, the initiation of cachectic immunological changes was observed prior to weight loss, during the pre-cachexia stage. Accordingly, these findings reveal that the monitoring of humoral and immunological factors may be more sensitive than weight loss for the initial diagnosis and treatment of cachexia.
\end{abstract}

Keywords: C26 tumor-bearing mice, Cancer cachexia, CD4+ T cell, Cytotoxic T-lymphocyte-associated antigen-4, Immunosuppression, Monocyte chemoattractant protein-1

\section{Introduction}

Cancer patients with progressive cachexia suffer severe morbidity and a high mortality rate $(\sim 20 \%)[1]$. Cachexia directly affects the survival rate by inducing the failure of normal metabolism, leading to conditions such as hypogonadism, insulin resistance, and inflammation [2]. Cancer cachexia is difficult to treat and may eventually lead to death.

A typical symptom of cachexia is weight loss due to the wasting of skeletal muscle and adipose tissue; initial cachexia diagnosis guideline focused on sarcopenia and

\footnotetext{
*Correspondence: amy3523@kirams.re.kr

${ }^{4}$ Radiation Non-clinical Center, Korea Institute of Radiological and Medical Sciences, Seoul 01812, Korea

Full list of author information is available at the end of the article
}

weight loss as cachectic diagnostic markers [3, 4]. Using the criteria of these typical symptoms, classical cachexia progression is divided into pre-cachexia, cachexia and refractory cachexia [5]. As the interest regarding cachexia diagnosis is increasing, an additional cachexia score has been suggested as a multiplicative diagnostic index that considers body weight loss, composition, physical performance, anorexia, quality of life, immunosuppression, inflammation, and metabolic disturbance [6]. Furthermore, the levels of tumor necrosis factor (TNF)- $\alpha$, and interleukin (IL)-6, pro-inflammatory cytokines, are significantly elevated in conditions of progressed cachexia $[7,8]$. However, lack of the characteristics depending on the state of cachexia research represents a major obstacle to overcoming cachexia. 
A number of studies have reported that cancer therapy by drug treatment and surgery is not typically successful against cancer accompanied by cachexia; cancer cachexia also promotes a poor prognosis through its immunosuppressive effects [9, 10]. Immunosuppression could occur when immune cells are reduced and/or affected by inhibitory signals such as immune checkpoints molecules including T-lymphocyte-associated antigen 4 (CTLA4) and programmed cell death protein 1 (PD-1) [11]. Immunotherapy against cancer has focused on overcoming the immunosuppression of immune cells by improving immunological activity and inhibiting these immune checkpoints $[12,13]$.

Early diagnostic factors for cachexia are required for the success of anticancer therapy in cancer patients with cachexia, to allow treatment before immunological collapse. However, the factors that may be suitable for the early diagnosis of cancer cachexia have yet to be fully understood. In the present study, C26 tumor-bearing mice, a well-characterized and typically used animal model for cancer cachexia, were used for this purpose. On cancer cachexia mouse model, physiological and immunological characterization was conducted during cachexia progression.

\section{Materials and methods \\ Cell culture}

The C26 colon cancer cell lines were purchased from CLS Cell Line Service GmbH (cryovial no., 400156, Eppelheim, Germany). The C26 cells were cultured in RPMI-1640 medium (Welgene, Seoul, Korea) containing $10 \%$ fetal bovine serum (FBS), $100 \mathrm{U} / \mathrm{ml}$ penicillin, and $100 \mu \mathrm{g} / \mathrm{ml}$ streptomycin. Cells were cultured in a humidified incubator with an atmosphere of $5 \% \mathrm{CO}_{2}$ at $37^{\circ} \mathrm{C}$.

\section{Animal experiments}

All animal study protocols were approved by the Institutional Animal Care and Use Committee of the Korean Institute of Radiological and Medical Sciences (Seoul, Korea; approval no., KIRAMS 2017-0073). Male 6-weekold $\mathrm{Balb} / \mathrm{c}$ mice were purchased from Orient Bio, Inc. (Seognam, Korea) and maintained in a laminar airflow cabinet with specific pathogen-free conditions. The C26 model was established by subcutaneous injection of $2 \times 10^{5}$ C26 cells into the right hind leg. Two different animal experiments were conducted: Firstly, a cachexia group $(n=10)$ and a normal group $(n=5)$ were monitored at 23 days for refractory cachexia experiment. Secondly, mice were randomly divided into 5 groups $(n=5$ for cachexia induced model and $\mathrm{n}=3$ for normal without cachexia) and sacrificed at 3, 7, 14, and 21 days after inoculation for time-dependent experiments. According to the classical cachexia diagnosis that $5 \%$ body weight loss is lead by cachexia progression [4], we terminated experiment at 21 days after $\mathrm{C} 26$ cell inoculation when body weight of mice decreased by $5 \%$.

Total body weight and tumor volume were measured three times per week. The tumor volume $(\mathrm{V})$ was calculated using the standard formula: $\mathrm{V}\left(\mathrm{mm}^{3}\right)=\pi / 6 \mathrm{x}$

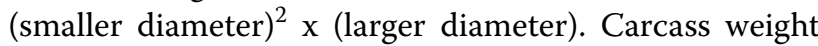
was calculated as the total body weight minus the tumor weight. Tumor weight was measured or calculated using the correlation factor between the actual tumor weight and its volume using the same methods in reference study [14]. At the end of each experiment, blood and spleen samples were obtained and the weights of the spleen, thymus, and liver were measured. All organ weights were expressed relative to the total body weight. The results were converted to fold-change compared with the value of normal control mice without cachexia.

\section{Isolation of single immune cells}

Spleen tissues samples were homogenized in PBS containing $1 \mathrm{mg} / \mathrm{ml}$ collagenase. The enzymatic reaction was terminated by adding $5 \mathrm{mM}$ EDTA. The homogenized tissue samples were minced on a 70- $\mu \mathrm{m}$ mesh grid cell strainer (SPL Life Sciences Co., Ltd., Pocheon, Korea). Red blood cells (RBCs) were removed using RBC lysis buffer (eBioscience, San Diego, CA, USA). The extracted single cells were washed with PBS containing $2 \%$ FBS, then stained with fluorescence-labeled antibodies for flow cytometry analysis as described in the subsequent section.

\section{Flow cytometric analysis}

Purified Rat Anti-Mouse CD16/CD32 (cat. no., 553142) was used to prevent non-specific binding on Fc-receptors prior to antibody staining for $15 \mathrm{~min}$ on ice for flow cytometry analysis. To identify the immune cell population, the following antibodies were used according to the manufacturer's protocols: BB515-conjugated rat anti-mouse I-A/I-E (cat. no., 564422) and allophycocyanin (APC)-cyanine (Cy)7-conjugated hamster anti-mouse CD11c (cat. no., 117324; BioLegend, San Diego, CA, USA) for dendritic cells (DCs); phycoerythrin (PE)-Cy7-conjugated hamster anti-mouse CD3e (cat. no., 552774), BB515-conjugated rat anti-mouse CD8a (cat. no., 564422), and APC-conjugated rat anti-mouse CD4 (cat. no., 553051) for T-cells; PE-cy7-conjugated hamster anti-mouse CD3e (cat. no., 552774) and PEconjugated mouse anti-mouse NK 1.1 (cat. no., 552878) for natural killer (NK) cells; PE-conjugated hamster anti-mouse CD152 (CTLA-4; cat. no., 553720) and peridinin-chlorophyll-protein (PerCP)/Cy5.5-conjugated anti-mouse CD279 (PD-1; cat. no., 135208; BioLegend, San Diego, CA, USA) for cell surface activation markers. 
Not annotated antibodies by manufactures were all from BD Biosciences, San Diego, CA, USA. Stained samples were evaluated on a FACSCalibur flow cytometer or FACSAccuri C6 flow cytometer (BD Biosciences) and data were analyzed using Flowjo software (version.10, FlowJo, LLC, Ashland, OR, USA). The gating strategy to identify immune cells population and immune checkpoints was as follows: (1) Splenocytes were gated in total $\mathrm{T}$ cell as $\mathrm{CD}^{+} \mathrm{T}$ cells, then divided into $\mathrm{CD} 4$ and $\mathrm{CD} 8^{+}$ $\mathrm{T}$ cells respectively, (2) the $\mathrm{CD} 4^{+} / \mathrm{CD}^{+}$population was further analyzed for the detection of CTLA-4 and PD-1, checkpoint surface markers, (3) NK cells were $\mathrm{CD}^{-}$ and NK $1.1^{+}$, while DC cells were I-A/I-E $\mathrm{E}^{+}$and CD $11 \mathrm{c}^{+}$ population.

\section{Cytokine analysis in blood serum}

Blood samples were centrifuged at $1500 \times g$ for $20 \mathrm{~min}$ to collect serum. Mouse serum cytokines were detected by ELISA using mouse Quantikine ELISA kits for IL-6 (cat. no., M6000B), TNF- $\alpha$ (cat. no., MHSTA50) and monocyte chemoattractant protein-1 (MCP-1; cat. no., MJE00; all from R\&D Systems, Minneapolis, MN, USA) according to the manufacturer's protocol.

\section{Statistical analysis}

All results are expressed as the mean \pm standard error of the mean. Statistical analysis was performed using an independent $t$ test or one-way analysis of variance followed by Tukey's significant difference test with SPSS software version 23.0 (IBM Corp., Armonk, NY, USA). $P<0.05$ was considered to indicate a statistically significant difference.

\section{Results}

\section{Cachectic characterization of refractory cachexia} in cachexia mouse model

Starting from 4 days after $\mathrm{C} 26$ cell inoculation, the tumor volume was measured 3 times per week. Cachexia progression in this study represented to scheme accompanying by international concensus reported by Fearon et al. [4] (Fig. 1a). As displayed in Fig. 1b, tumor volume steadily increased in a time-dependent manner. At 23 days after inoculation, the mean body weight in the cachexia group was markedly lower $(0.83 \pm 0.02$-fold $)$ than that in the normal control group $(P<0.001$; Fig. 1 c). In organ weight changes, spleen weight in the cachexia group increased by $2.94 \pm 0.13$-fold $(P<0.01)$, while the liver

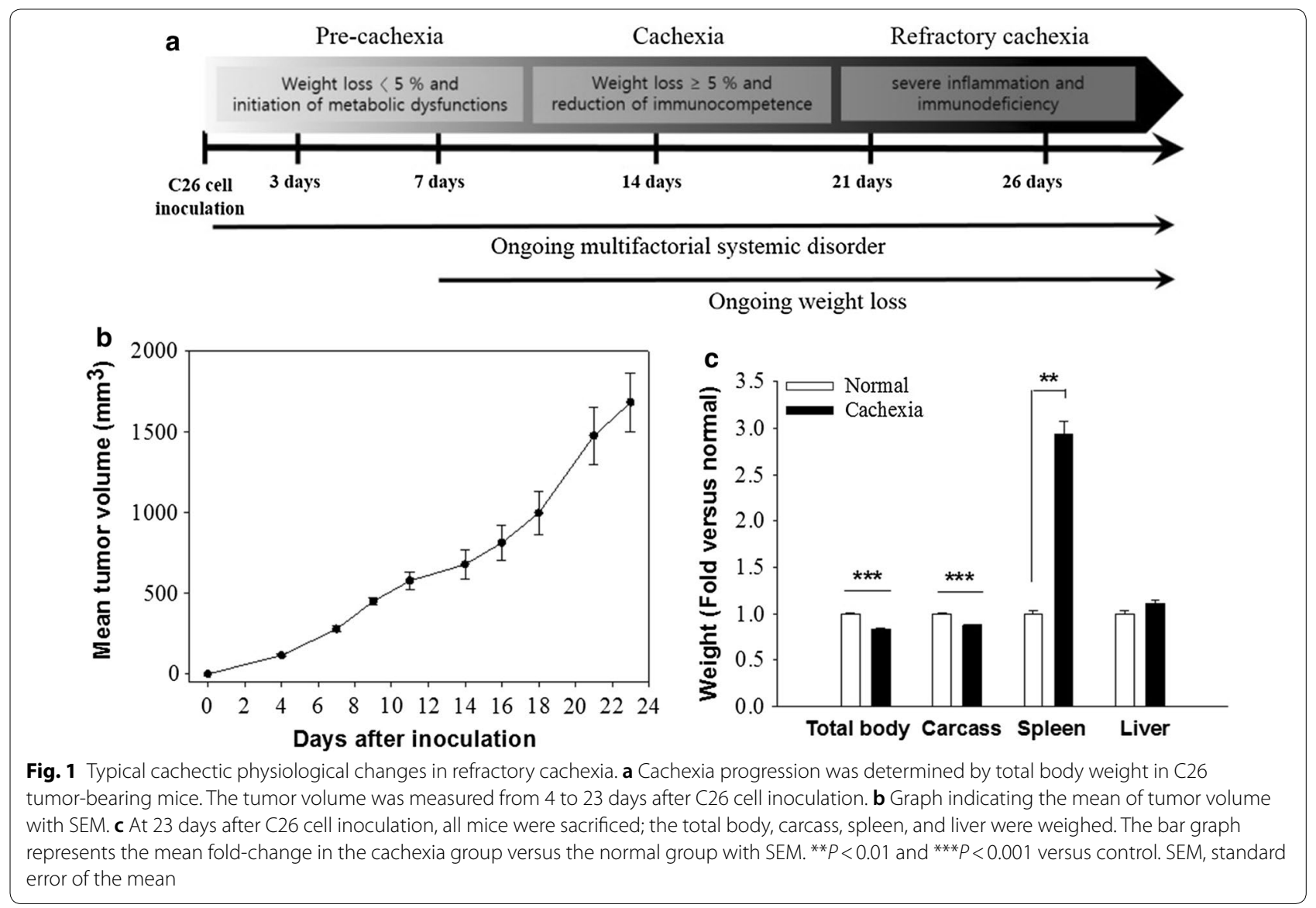


weight was not significantly altered, compared with the normal group (Fig. 1c).

\section{Analysis of immune cell populations and immune checkpoints of refractory cachexia in cachexia mouse model}

The numbers of T cells, NK cells, and DCs in spleen were measured by flow cytometry. The populations of $\mathrm{CD}^{+}$, $\mathrm{CD}^{+}$, and $\mathrm{CD}^{+}{ }^{+} \mathrm{T}$ cells in the spleens from the cachexia group were significantly lower $(0.71 \pm 0.04-, 0.70 \pm 0.04-$, and $0.75 \pm 0.06$-fold, respectively) than in the normal group (all $P<0.05$ ), while the NK cell and DC populations were unchanged (Fig. 2a). The expression of CTLA-4, an early-reactive immune checkpoint marker, and PD-1, a late-reactive immune checkpoint marker, on $\mathrm{CD}_{4}^{+}$ and $\mathrm{CD}^{+} \mathrm{T}$ cells was also examined. The expression of immune checkpoint markers on cells from the cachexia group was lower than in the normal group (Fig. 2b). These results demonstrate that $\mathrm{T}$ cells were depleted to a greater extent than NK and DC cells during in refractory cachexia, whereas no immunosuppression by activation of immune checkpoint markers on T cells was observed.

\section{Changes in total body, carcass, organ weights, and cytokine changes during cachexia progression}

The total body, carcass, and organ weights were determined at 3, 7, 14, and 21 days from C26 cell inoculation. The expected tumor weights were calculated based on the tumor volumes (Fig. 3a). At 21 days after C26 cell inoculation, the mean total body weight of the cachexia group decreased significantly compared with the normal group $(P<0.01)$. In particular, the carcass weight of cachectic mice consistently decreased from 10 days after C26 cell inoculation compared with the normal group $(P<0.01$; Fig. $3 \mathrm{~b})$. Therefore, the carcass weight, i.e. the body weight minus the tumor weight, in the cachexia group was significantly reduced from 10 to 21 days after C26 cell inoculation.

Additionally, changes in organ weights, thymus weights were significantly decreased at $21(P<0.05)$ compared with 3 days from $\mathrm{C} 26$ cell inoculation. In particular, the thymus weight was significantly reduced $(0.35 \pm 0.12$ fold) at 21 days from C26 cell inoculation compared with the normal group (Fig. 3c). In contrast, spleen weight was markedly increased at 14 and 21 days compared with 3 days from $\mathrm{C} 26$ cell inoculation $(P<0.01$; Fig. $3 c)$. At 14 days after $\mathrm{C} 26$ cell inoculation, the spleen weight reached a maximum, and then slightly decreased thereafter. The spleen weight increased in pre-cachexia, but decreased in refractory cachexia. These are that one of the classical cachexia symptoms is unexpected wasting symptom like losing body composition. Thus, the decrease of spleen weight seemed to be resulted from

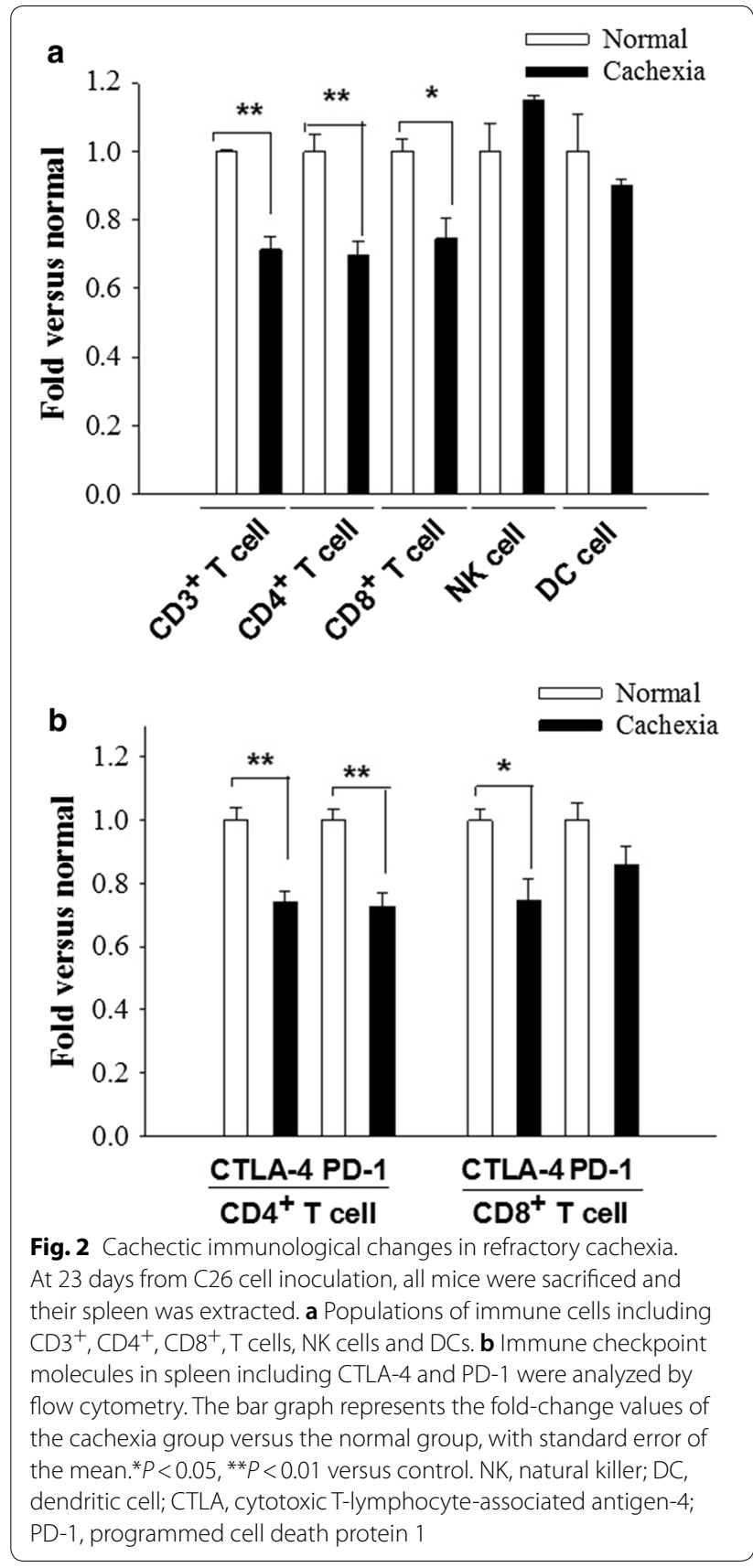

cachextic wasting of body composition. The liver weight was not significantly different compared with the normal group.

The levels of inflammatory cytokines including IL-6, TNF- $\alpha$, and MCP-1, were determined in the blood serum at $3,7,14$, and 21 days from C26 cell inoculation. Of the cytokines assessed, the respective levels of MCP-1 and IL- 6 in the cachexia group were increased by $1.84 \pm 0.16$ and $3.63 \pm 0.25$-fold at 3 days from $\mathrm{C} 26$ cell inoculation compared with the normal group. TNF- $\alpha$ and MCP-1 

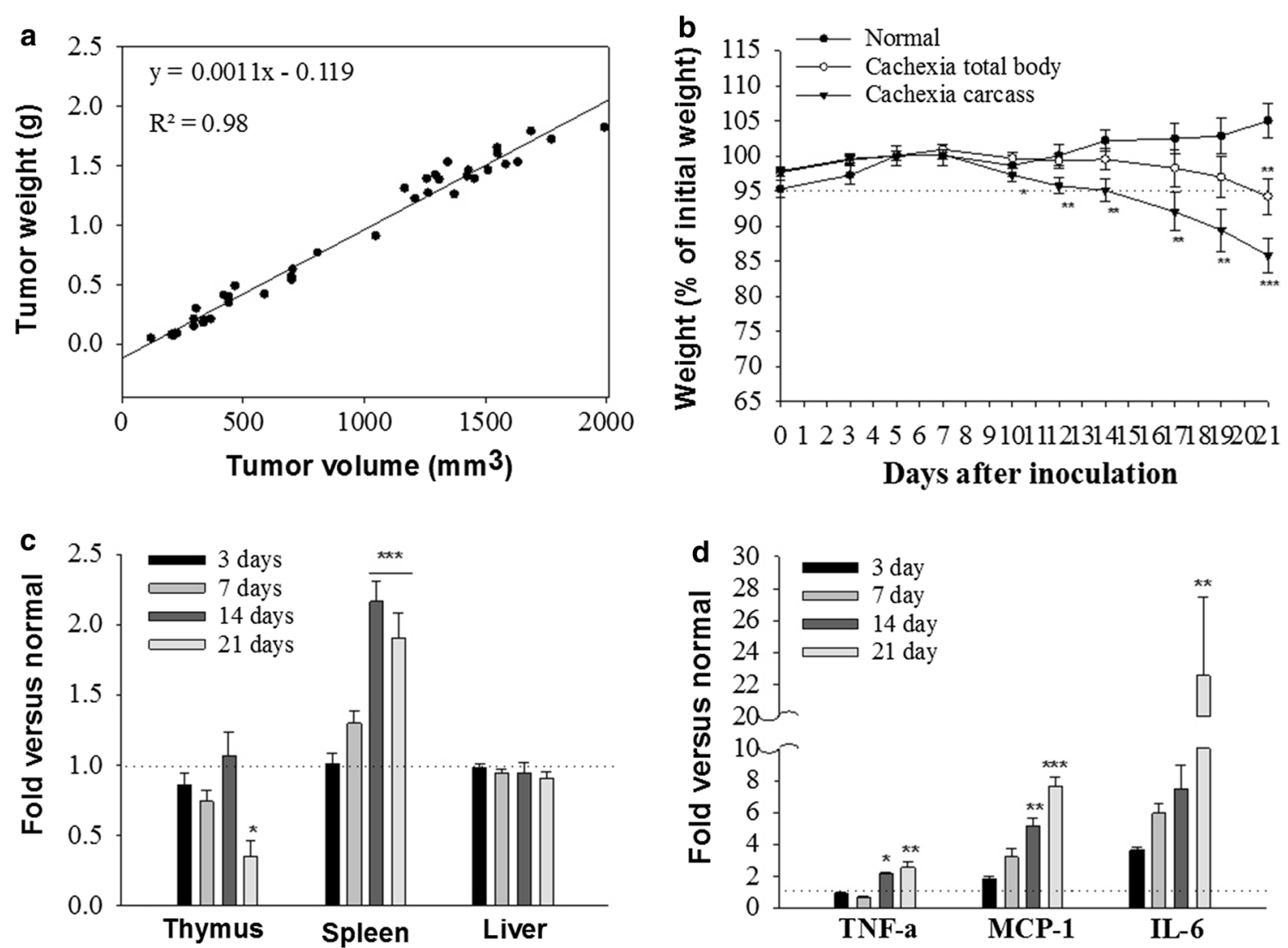

Fig. 3 Physiological changes during cachexia progression. For physiological characterization during cachexia progression, the total body, carcass, organs weights, and cytokine change were monitored at 3, 7, 14, and 21 days after C26 cell inoculation. a Using the equation for estimating tumor weight from tumor volume obtained from a previous study, $\mathbf{b}$ the graph indicates the percentage of total body and carcass weight with S.E.M in the normal and cachexia groups. Dotted lines indicate $5 \%$ weight loss, indicating the end of the pre-cachexia stage. ${ }^{*} P<0.05,{ }^{* *} P<0.01,{ }^{* * *} P<0.001$ versus control. c The thymus, spleen, and liver were weighed. The bar graph represents fold-change values in the cachexia group versus the normal group, with SEM. Dotted lines indicate the normal group fold-change. ${ }^{* *} P<0.01,{ }^{* *} P<0.001$ versus 3 days after C26 cell inoculation. $\mathbf{d}$ Analysis of inflammatory cytokines during cachexia progression in blood serum samples. The levels of inflammatory cytokines were measured using an ELISA kit. The bar graph represents fold-change values of the cachexia group versus the normal group with standard error of the mean. Dotted lines indicate the normal group fold-change. ${ }^{*} P<0.05,{ }^{* *} P<0.01$, and ${ }^{* *} P<0.001$ versus 3 days after $C 26$ cell inoculation. IL, interleukin; TNF, tumor necrosis factor; $M C P$, monocyte chemoattractant protein-1

levels were significantly increased at 14 and 21 days compared with 3 days after $\mathrm{C} 26$ cell inoculation $(P<0.05)$. In particular, the level of IL-6 was $22.5 \pm 6.34$-fold higher at 21 days after $\mathrm{C} 26$ cell inoculation than the normal group, representing a significant increase relative to 3 days after C26 cell inoculation $(P<0.01$; Fig. 3d).

\section{Analysis of immune cell populations and immune checkpoints during cancer cachexia progression}

Changes in the immune cell population of spleen during cancer cachexia progression were analyzed by flow cytometry. Starting from 14 days after C26 cell inoculation, the populations of $\mathrm{CD}^{+}$, helper $\mathrm{CD}_{4}^{+}$, and cytotoxic $\mathrm{CD} 8^{+} \mathrm{T}$ cells were significantly reduced compared with 3 days after $\mathrm{C} 26$ cell inoculation $(P<0.01$; Fig. $4 a)$. The critical time at which changes in the immune response in cancer cachexia model occurred was 14 days from $\mathrm{C} 26$ cell inoculation.

Next, the changes in immune checkpoints over time were investigated. In $\mathrm{CD} 4^{+} \mathrm{T}$ cells, CTLA- 4 expression in the cachexia group was increased by $1.45 \pm 0.02$-fold at 7 days from $C 26$ cell inoculation compared with at 3 days $(P<0.01)$. The PD- 1 expression on $\mathrm{CD}^{+} \mathrm{T}$ cells was significantly increased at 21 days compared with 3 days after $\mathrm{C} 26$ cell inoculation $(P<0.01)$. For $\mathrm{CD} 8^{+}$ $\mathrm{T}$ cells, CTLA-4 expression was markedly increased at 21 days compared with at 3 days after $\mathrm{C} 26$ cell inoculation $(P<0.01)$, but no change in PD-1 expression was observed compared with the normal or cachexia 


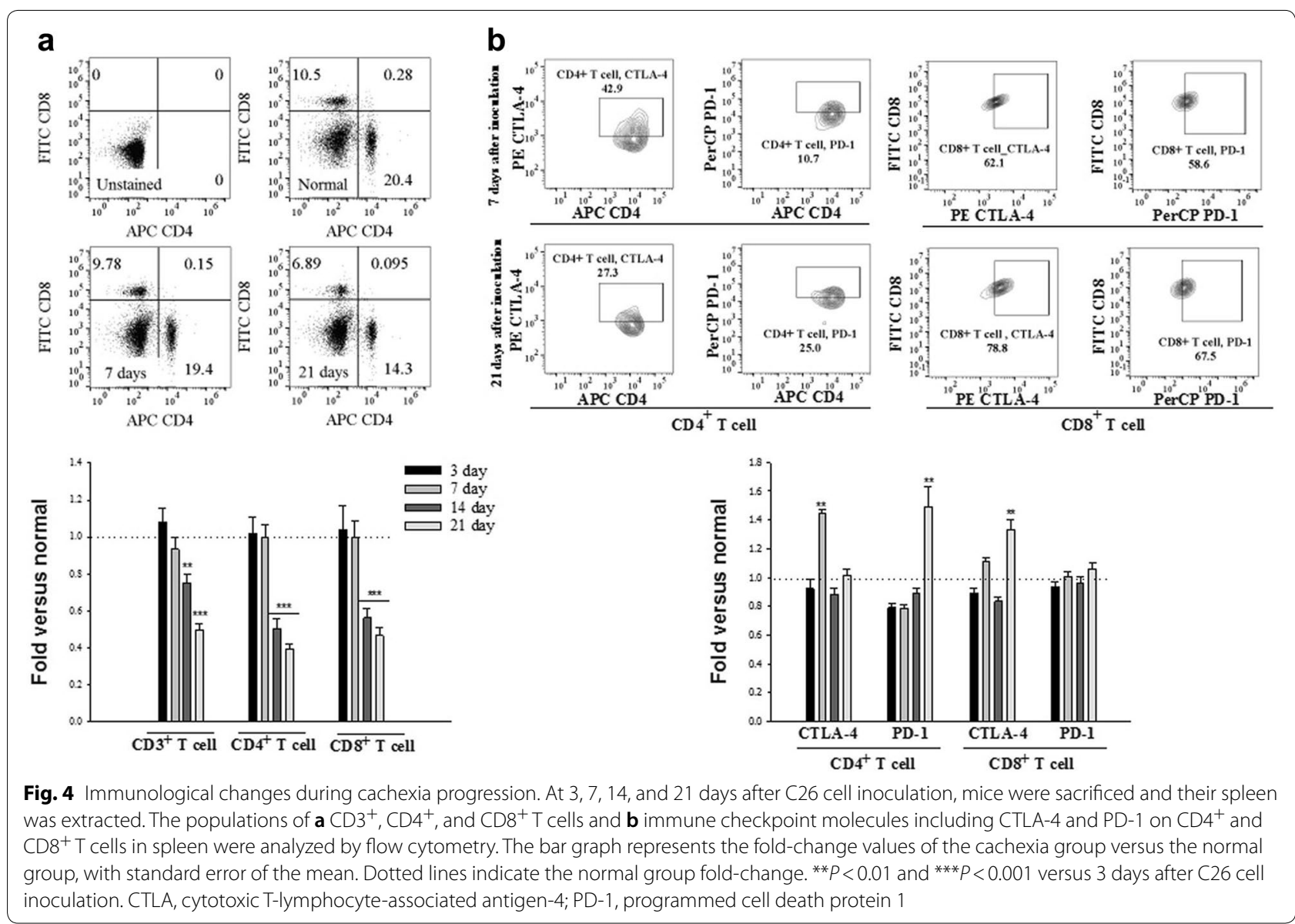

progression groups (Fig. 4b). Thus, changes in CTLA-4 and PD-1 expression during cachexia progression occurred more rapidly in $\mathrm{CD}^{+}$cells than in $\mathrm{CD} 8^{+}$ cells.

\section{Discussion}

The incidence of cachexia is highest in patients with incurable gastrointestinal cancer. Teunissen et al. [15] reported that the prevalence of cachectic weight loss in patients with cancer increases from 46 to $86 \%$ in the last 1-2 weeks of a patient's life. Because of the progressive weight loss, cachectic symptoms reduce a patient's tolerance to cancer treatment [16]. In order to understand cachexia metabolism, recent studies have focused on the mechanism of wasting of skeletal muscle and fat tissue, and immunological metabolism [17, 18]. However, trials to block or relieve cachectic wasting are rarely successful, and the understanding of cachexia metabolism remains incomplete. From difficulties in assessment with wellknown but, irregular cachectic dysfunction on cachexia, we hypothesize that there is a critical failure in the host metabolism prior to the wasting phenomenon. Thus, in the present study, time-dependent cachectic dysfunction was examined through monitoring physiological and immunological changes during cachexia progression.

Based on these confirmatory results in the refractory cachexia stage, the time-dependent dysfunction of the host metabolism and immune system associated with cachexia progression was examined. With regards to physiological characterization during cachexia progression, the total body, carcass, and organs weights were monitored over time. Notably, the initiation of changes in the spleen, a major immune organ, was observed in the pre-cachexia stage. The results further indicated that cachectic splenomegaly and thymus atrophy were induced in a time-dependent manner (Fig. 3c). Splenomegaly commonly occurs in tumor cell transplantable models, including in C26 colon cancer model mice [19-21]. In normal conditions, the spleen is the site of myeloid cell differentiation including macrophages, DCs, and immune cells with innate and adaptive immune defense functions [19]. However, in oncogenesis, myeloid cells are affected by humoral factors and cell-to-cell interactions in the tumor-environment, and may be differentiated into myeloid-derived suppressor cells in tumor-bearing animals. Numerous studies have 
Table 1 Summary of immunological and physiological characterization during cachexia progression though our findings

\begin{tabular}{|c|c|c|c|c|}
\hline \multirow[t]{3}{*}{ Characteristics } & \multicolumn{2}{|c|}{ Pre-cachexia } & \multirow[t]{2}{*}{ Cachexia } & \multirow[t]{2}{*}{ Refractory cachexia } \\
\hline & \multicolumn{2}{|c|}{ Days after C26 cell inoculation } & & \\
\hline & 3 days & 7 days & 14 days & 21 days \\
\hline \multicolumn{5}{|l|}{ Immunological related } \\
\hline \multicolumn{5}{|l|}{ T cell populations } \\
\hline Total T cell (CD3 positive) & N.C. & N.C. & $\downarrow$ & $\downarrow \downarrow \downarrow$ \\
\hline Helper T cell (CD4 positive) & N.C. & N.C. & $\downarrow \downarrow \downarrow$ & $\downarrow \downarrow \downarrow$ \\
\hline Cytotoxic T cell (CD8 positive) & N.C. & N.C. & $\downarrow \downarrow \downarrow$ & $\downarrow \downarrow \downarrow$ \\
\hline \multicolumn{5}{|l|}{ Immune checkpoints } \\
\hline \multicolumn{5}{|l|}{ CTLA-4 } \\
\hline on $\mathrm{CD}^{+}{ }^{+} \mathrm{T}$ cell & N.C. & $\uparrow \uparrow$ & N.C. & N.C. \\
\hline on $\mathrm{CD}^{+} \mathrm{T}$ cell & N.C. & N.C. & N.C. & $\uparrow \uparrow$ \\
\hline \multicolumn{5}{|l|}{ PD-1 } \\
\hline on $\mathrm{CD}^{+}{ }^{+} \mathrm{T}$ cell & N.C. & N.C. & N.C. & $\uparrow \uparrow$ \\
\hline on $\mathrm{CD}^{+} \mathrm{T}$ cell & N.C. & N.C. & N.C. & N.C. \\
\hline \multicolumn{5}{|l|}{ Cytokines } \\
\hline TNF-a & N.C. & N.C. & $\uparrow$ & $\uparrow \uparrow$ \\
\hline MCP-1 & $\hat{i}$ & $\uparrow$ & $\uparrow \uparrow$ & $\uparrow \uparrow \uparrow$ \\
\hline IL-6 & $\uparrow$ & $\hat{\imath}$ & $\hat{\imath}$ & $\uparrow \uparrow \uparrow$ \\
\hline \multicolumn{5}{|l|}{ Physiological related } \\
\hline Carcass weight & N.C. & N.C. & $\downarrow$ & $\downarrow \downarrow \downarrow$ \\
\hline \multicolumn{5}{|l|}{ Organs } \\
\hline Thymus & N.C. & N.C. & N.C. & $\downarrow$ \\
\hline Spleen & N.C. & $\uparrow$ & $\uparrow \uparrow \uparrow$ & $\uparrow \uparrow \uparrow$ \\
\hline Liver & N.C. & N.C. & N.C. & N.C. \\
\hline
\end{tabular}

N.C.: no change, arrow with dotted line: high tendency compared with normal group, arrow with solid line: significance compared with 3 days after C26 cell inoculation, 1 of arrow with solid line: $P<0.05,2$ of arrow with solid line: $P<0.01,3$ of: arrow with solid line: $P<0.001$

reported that myeloid-derived suppressor cells, which express immunosuppressive enzymes, accumulate in the spleen and tumor [22]. As a result of immune reactions in oncogenesis, spleen volume and weight may increase due to massive granulocytic infiltration of red pulp [23]. Accordingly, splenomegaly in a cachexia animal model may represent an immunosuppressive reaction, but this requires further analysis.

In addition, thymus atrophy was markedly induced at 21 days after $\mathrm{C} 26$ cell inoculation in the cachexia group (Fig. 3c). The thymus is responsible for producing $\mathrm{T}$ cell progenitors, crucial immunocompetent cells. Numerous clinical studies have reported on the relationships between the thymus, $\mathrm{T}$ cells and immunodeficiency. Patients suffering from immunodeficiency symptoms due to DiGeorge or Down's syndrome have little or no thymus and show decreased naïve $\mathrm{T}$ cell levels $[24,25]$. According that, thymus atrophy and $\mathrm{T}$ cell number reduction are associated with the immunodeficiency symptom of cachexia, the same phenomenon of thymus atrophy followed by $\mathrm{T}$ cell reduction results was experimentally confirmed in the present study.
To analyze the immunological characterization during cachexia progression, we assessed the $\mathrm{T}$ cell populations and immune checkpoint molecule levels over time in the spleen. Starting 14 days from $\mathrm{C} 26$ cell inoculation, $\mathrm{CD}^{+}$, $\mathrm{CD}^{+}$, and $\mathrm{CD}^{+} \mathrm{T}$ cell populations in the cachexia group were significantly reduced compared with the normal group (Fig. 4a). These results demonstrate that changes in the spleen, thymus and $\mathrm{T}$ cell populations occurred in pre-cachexia stage, becoming more severe in a timedependent manner. Furthermore, depending on the $\mathrm{T}$ cell type during cachexia progression, the expression of CTLA-4, an early-reactive immune checkpoint marker, was increased on $\mathrm{CD} 4^{+} \mathrm{T}$ cells at 7 days and $\mathrm{CD} 8^{+} \mathrm{T}$ cells at 21 days. Additionally, the expression of PD-1, a latereactive immune checkpoint marker, was only increased on $\mathrm{CD}^{+} \mathrm{T}$ cells at 21 days after $\mathrm{C} 26$ cell inoculation, but not on $\mathrm{CD}^{+} \mathrm{T}$ cells. The analysis of immune checkpoint molecules revealed that immunosuppression by activation of CTLA-4 in the cachexia group was induced earlier on $\mathrm{CD}^{+}$than on $\mathrm{CD}^{+} \mathrm{T}$ cells. Wang et al. [26] reported that $\mathrm{CD}^{+}$sub-populations delayed the onset of wasting symptoms in diabetes and reduced muscle atrophy 
in cachexia; the $\mathrm{CD} 4^{+} \mathrm{T}$ cell population appeared to be closely associated with cachectic dysfunction, similar to the conclusion of the present study. Therefore, to further confirm the immunological characterization of cachexia progression, early-reactive $\mathrm{CD} 4^{+} \mathrm{T}$ cells and their surface markers in the spleen to allow the early initial diagnosis of cachexia require further study.

In the cytokine analysis results, consistent with previous studies $[5,17,27,28]$, the classical cachectic cytokines TNF- $\alpha$ and IL- 6 were also increased in cachexic condition. Notably, in the pre-cachexia stage, the MCP-1 and IL- 6 levels in the cachexia group were highly increased at 3 days after $\mathrm{C} 26$ cell inoculation compared with the normal group (Fig. 3d). Talbert et al. [29] reported that clinically, the MCP-1 level is more associated with the earlier stages of cachexia than the classical cachetic cytokines. In accord with that study, the results of the present study also suggest that MCP-1 may be useful in the early diagnosis of cachexia. The physiological and immunological characterizations of cachexia progression were summarized (Table 1). Changes in immunological factors including reduced and altered $\mathrm{T}$ cell populations, activated immune checkpoints and elevated cachectic cytokine levels, manifested in the pre-cachexia stage when $<5 \%$ loss of total body weight had occurred. Changes in MCP-1 level and immune checkpoint expression on $\mathrm{CD} 4^{+} \mathrm{T}$ cells from the spleen were reported for the first time. Taken together, our results suggest that $\mathrm{CD}^{+}{ }^{+} \mathrm{T}$ cells and MCP-1 may be useful for initial diagnosis of cachexia more accurately than total body or carcass weight changes. Moreover, these results will contribute to providing a scientific rationale for the early diagnosis of cachexia and cachexia treatment.

\section{Authors' contributions}

YKJ and JEJ conceived and designed the experiments. JEJ, JYL, and MSL performed the experiments. JEJ, YKJ, and M-SK analyzed the data. YKJ, JHK, EHK, and NC contributed reagents/materials/analysis tools. JEJ and YKJ wrote the manuscript.

\section{Author details \\ ${ }^{1}$ Department of Biosystems Engineering, College of Life Sciences and Bio- technology, Korea University, Seoul 02841, Korea. ${ }^{2}$ RI Applied Research Team, Division of Applied RI, Korea Institute of Radiological and Medical Sciences, Seoul 01812, Korea. ${ }^{3}$ Department of Radiation Oncology, Korea Institute of Radiological and Medical Sciences, Seoul 01812, Korea. ${ }^{4}$ Radia- tion Non-clinical Center, Korea Institute of Radiological and Medical Sciences, Seoul 01812, Korea. ${ }^{5}$ Radiation Molecular Diagnosis Research Team, Division of Radiation Biomedical Research, Korea Institute of Radiological and Medical Sciences, Seoul 01812, Korea.}

\section{Acknowledgements}

This research was supported by the National Research Foundation of Korea, funded by the Ministry of Education (Grant No., NRF-2017R1D1A1B03031982) and the Industrial Core Technology Development Program (Grant No., 10051129; Development of the system for ADME assessment using radiolabeled compounds) funded by the Ministry of Trade, Industry \& Energy (Republic of Korea).

\section{Competing interest}

The authors declare that they have no competing interests.

\section{Publisher's Note}

Springer Nature remains neutral with regard to jurisdictional claims in published maps and institutional affiliations.

Received: 26 November 2018 Accepted: 31 January 2019

Published online: 21 February 2019

\section{References}

1. Fearon KC, Glass DJ, Guttridge DC (2012) Cancer cachexia: mediators, signaling, and metabolic pathways. Cell Metab 16:153-166

2. Evans WJ, Morley JE, Argiles J et al (2008) Cachexia: a new definition. Clin Nutr 27:793-799

3. Tisdale MJ (1999) Wasting in cancer. J Nutr 129:243S-246S

4. Fearon K, Strasser F, Anker SD et al (2011) Definition and classification of cancer cachexia: an international consensus. Lancet Oncol 12:489-495

5. Vaughan VC, Martin P, Lewandowski PA (2013) Cancer cachexia: impact, mechanisms and emerging treatments. J Cachexia Sarcopenia Muscle 4:95-109

6. Argiles JM, Lopez-Soriano FJ, Toledo M et al (2011) The cachexia score (CASCO): a new tool for staging cachectic cancer patients. J Cachexia Sarcopenia Muscle 2:87-93

7. Fogelman DR, Morris J, Xiao L et al (2017) A predictive model of inflammatory markers and patient-reported symptoms for cachexia in newly diagnosed pancreatic cancer patients. Support Care Cancer 25:1809-1817

8. Taksande BG, Gawande DY, Chopde CT et al (2017) Agmatine ameliorates adjuvant induced arthritis and inflammatory cachexia in rats. Biomed Pharmacother 86:271-278

9. Muscaritoli M, Bossola M, Aversa Z et al (2006) Prevention and treatment of cancer cachexia: new insights into an old problem. Eur J Cancer 42:31-41

10. Evans C, Dalgleish AG, Kumar D (2006) Review article: immune suppression and colorectal cancer. Aliment Pharmacol Ther 24:1163-1177

11. Pardoll DM (2012) The blockade of immune checkpoints in cancer immunotherapy. Nat Rev Cancer 12:252-264

12. Ayeka PA, Bian Y, Githaiga PM et al (2017) The immunomodulatory activities of licorice polysaccharides (Glycyrrhiza uralensis Fisch.) in CT 26 tumor-bearing mice. BMC Complement Altern Med 17:536

13. Coelho MA, de Carne Trecesson S, Rana S et al (2017) Oncogenic RAS signaling promotes tumor Immunoresistance by stabilizing PD-L1 mRNA. Immunity 47(1083-1099):e1086

14. Tanaka Y, Eda H, Tanaka T et al (1990) Experimental cancer cachexia induced by transplantable colon 26 adenocarcinoma in mice. Cancer Res 50:2290-2295

15. Teunissen SC, Wesker W, Kruitwagen C et al (2007) Symptom prevalence in patients with incurable cancer: a systematic review. J Pain Symptom Manag 34:94-104

16. Ross PJ, Ashley S, Norton A et al (2004) Do patients with weight loss have a worse outcome when undergoing chemotherapy for lung cancers? Br J Cancer 90:1905-1911

17. Porporato PE (2016) Understanding cachexia as a cancer metabolism syndrome. Oncogenesis 5:e200

18. Bruggeman AR, Kamal AH, LeBlanc TW et al (2016) Cancer cachexia: beyond weight loss. J Oncol Pract 12:1163-1171

19. Gabrilovich DI, Ostrand-Rosenberg S, Bronte V (2012) Coordinated regulation of myeloid cells by tumours. Nat Rev Immunol 12:253-268

20. Aulino P, Berardi E, Cardillo VM et al (2010) Molecular, cellular and physiological characterization of the cancer cachexia-inducing C26 colon carcinoma in mouse. BMC Cancer 10:363

21. Bonetto A, Rupert JE, Barreto R et al (2016) The colon-26 carcinoma tumor-bearing mouse as a model for the study of cancer cachexia. J Vis Exp 117:e54893. https://doi.org/10.3791/54893

22. Bronte V, Pittet MJ (2013) The spleen in local and systemic regulation of immunity. Immunity 39:806-818 
23. DuPre SA, Hunter KW Jr (2007) Murine mammary carcinoma 4T1 induces a leukemoid reaction with splenomegaly: association with tumor-derived growth factors. Exp Mol Pathol 82:12-24

24. Gui J, Mustachio LM, Su DM et al (2012) Thymus size and age-related thymic involution: early programming, sexual dimorphism, progenitors and stroma. Aging Dis 3:280-290

25. Itoh S, Ohno T, Kakizaki S et al (2011) Epstein-Barr virus-positive T-cell lymphoma cells having chromosome 22q11.2 deletion: an autopsy report of DiGeorge syndrome. Hum Pathol 42:2037-2041

26. Wang Z, Zhao C, Moya R et al (2008) A novel role for CD4 ${ }^{+} \mathrm{T}$ cells in the control of cachexia. J Immunol 181:4676-4684
27. Camargo RG, Riccardi DM, Ribeiro HQ et al (2015) NF-kappaBp65 and expression of its pro-inflammatory target genes are upregulated in the subcutaneous adipose tissue of cachectic cancer patients. Nutrients 7:4465-4479

28. Morley JE, Thomas DR, Wilson MM (2006) Cachexia: pathophysiology and clinical relevance. Am J Clin Nutr 83:735-743

29. Talbert EE, Lewis HL, Farren MR et al (2018) Circulating monocyte chemoattractant protein-1 (MCP-1) is associated with cachexia in treatmentnaive pancreatic cancer patients. J Cachexia Sarcopenia Muscle. https:// doi.org/10.1002/jcsm.12251

\section{Submit your manuscript to a SpringerOpen ${ }^{\circ}$ journal and benefit from:}

- Convenient online submission

- Rigorous peer review

- Open access: articles freely available online

- High visibility within the field

- Retaining the copyright to your article

Submit your next manuscript at $\boldsymbol{\nabla}$ springeropen.com 